\title{
An Age-Dependent Change in the Set Point of Synaptic Homeostasis
}

\author{
Rebekah E. Mahoney, ${ }^{1}$ Joel M. Rawson, ${ }^{1,2}$ and Benjamin A. Eaton ${ }^{1,2}$ \\ ${ }^{1}$ Department of Physiology and ${ }^{2}$ Barshop Institute of Longevity and Aging Studies, University of Texas Health Sciences Center at San Antonio, San Antonio, \\ Texas 78229
}

Homeostatic plasticity functions within the nervous system to maintain normal neural functions, such as neurotransmission, within predefined optimal ranges. The defined output of these neuronal processes is referred to as the set point, which is the value that the homeostatic system defends against fluctuations. Currently, it is unknown how stable homeostatic set points are within the nervous system. In the present study we used the CM9 neuromuscular junctions (NMJs) in the adult Drosophila to investigate the stability of the set point of synaptic homeostasis across the lifespan of the fly. At the fly NMJ, it is believed that the depolarization of the muscle by neurotransmitter during an action potential, represented by the EPSP, is a homeostatic set point that is precisely maintained via changes in synaptic vesicle release. We find that the amplitude of the EPSP abruptly increases during middle age and that this enhanced EPSP is maintained into late life, consistent with an age-dependent change to the homeostatic set point of the synapse during middle age. In support of this, comparison of the homeostatic response at the young versus the old synapse shows that the magnitude of the homeostatic response at the older synapse is significantly larger than the response at the young NMJ, appropriate for a synapse at which the set point has been increased. Our data demonstrate that the amplitude of the EPSP at the Drosophila NMJ increases during aging and that the homeostatic signaling system adjusts its response to accommodate the new set point.

Key words: neurotransmission; homeostasis; aging; set point; neuromuscular junction; Drosophila

\section{Introduction}

Many regulated biological systems use homeostatic control mechanisms to maintain operating ranges for optimal performance. A hallmark of a homeostatic signaling system is the constant maintenance of a precisely defined output, referred to as the "set point." When challenged, the homeostatic system will respond in a compensatory fashion to reestablish the set point level of output. Within the nervous system there appears to be a number of essential neuronal functions, such as neuronal excitability, neuronal firing rates, and synapse function, whose output appears to defined by a homeostatic set point (Turrigiano et al., 1994; Marder and Prinz, 2003; Turrigiano and Nelson, 2004; Davis, 2006). The mechanisms that define the homeostatic set points within the nervous system are unclear, but existing data suggest that they may represent an intrinsic property of the neuron that emerges during development (Turrigiano et al., 1995; Thoby-

Received Aug. 20, 2013; revised Dec. 7, 2013; accepted Dec. 11, 2013.

Author contributions: R.E.M., J.M.R., and B.A.E. designed research; R.E.M. and J.M.R. performed research; R.E.M., J.M.R., and B.A.E. analyzed data; R.E.M. and B.A.E. wrote the paper.

This work was supported by an R01 Award (NS062811) and an Ellison Young Scholar Award (AG-NS-0415-07) to B.A.E. J.M.R. was supported by Grant T32-AG021890 from the National Institute on Aging. We thank Tabita Kreko and Jorge Azpurua for comments on earlier versions of this manuscript, and Graeme Davis for discussions about the data.

Correspondence should be addressed to Benjamin A. Eaton, Department of Physiology, University of Texas Health Sciences Center at San Antonio, San Antonio, TX 78229. E-mail: eatonb@uthscsa.edu.

J. M. Rawson's present address: Department of Biological Sciences, University of Alaska Anchorage, Anchorage, AK 99508.

DOI:10.1523/JNEUROSCI.3556-13.2014

Copyright $\odot 2014$ the authors $\quad 0270-6474 / 14 / 342111-09 \$ 15.00 / 0$
Brisson and Simmers, 2000; Moody and Bosma, 2005; McCabe et al., 2006; Marie et al., 2010; Marder and Prinz, 2002; Davis, 2013). Although most data support the notion that set points are extremely stable, changes to neuronal functions during certain pathologic conditions, such as aging, suggest that set points might be adjustable. To date, there is little experimental evidence demonstrating changes in homeostatic set points within the nervous system under any condition.

A hallmark of aging is the widespread decline in nervous system function, which includes deterioration of both motor performance and cognitive functions. Existing data support the concept that an important contributor to the declines in neural function during aging is synaptic dysfunction arising from altered neurotransmission (Kelly, 1978; Landfield et al., 1978; Hori et al., 1992; Robbins, 1992; Foster, 2007; Morrison and Baxter, 2012). In addition, many aspects of synapse function, both presynaptically and postsynaptically, are known to be under homeostatic control (Davis et al., 1998; Turrigiano et al., 1998; Burrone et al., 2002). Thus, it is unclear what role, if any, homeostatic signaling plays during changes in nervous system function with age. It is possible that homeostatic mechanisms are engaged during aging but are insufficient to overcome the age-dependent changes to neural function, or perhaps the homeostat is not designed to respond to age-dependent changes in function. Another possibility is that homeostatic signaling systems are not involved in the changes in synapse function observed with age but are, rather, intact and functioning normally throughout the lifespan of the animal. 
To address these questions, we have analyzed the effects of increasing age on the homeostatic set point of the synapse using the CM9 neuromuscular junction (NMJ) localized on the proboscis of the Drosophila fruit fly (Rawson et al., 2012). Using this system, we show that the synaptic homeostatic set point, represented at the Drosophila NMJ as the EPSP, abruptly increases during aging to an enhanced level that is stably maintained. Consistent with this interpretation, we find that the homeostatic response is significantly increased at older synapses compared with young synapses. These data provide evidence that the stable increase in EPSP amplitude observed during aging includes a change to the synaptic homeostatic set point.

\section{Materials and Methods}

Fly stocks. All fly stocks were maintained on a standard laboratory diet (Bloomington Stock Center recipe). All analysis was performed on virgin female flies that were flipped to freshly made food vials every other day and kept at 50\% humidity on a $12 \mathrm{~h}$ light/dark cycle (Rawson et al., 2012). Our wild-type stock is an isogenized laboratory stock of the $w^{1118}$ line (Rawson et al., 2012). The ephexin ${ }^{01953}$ allele was a gift from Dr. Andrew Frank (University of Iowa, Iowa City, IA) and backcrossed 5 times to our $w^{1118}$ stock before analysis.

CM9 NMJ microscopy. Immunofluorescent analysis of CM9 innervation was performed as previously described (Rawson et al., 2012). Briefly, female flies of the indicated age were anesthetized under carbon dioxide and decapitated. The head was transferred to a Sylgard-coated dissecting dish containing a small amount of ice-cold dissecting solution and then pinned with the proboscis extended. The posterior portion of the head was then dissected away and the remaining head and proboscis tissues were transferred to a $1.5 \mathrm{ml}$ tube and fixed at room temperature for 10 min in $4 \%$ paraformaldehyde in PBS/0.1\% Triton X-100 (TX-100). The fixed proboscis was then washed and blocking was performed with $0.1 \%$ BSA in PBS $/ 0.1 \%$ TX-100 for $1 \mathrm{~h}$ at room temperature. Primary antibodies to Discs-large (Dlg; 1:100; Developmental Studies Hybridoma Bank) and Drosophila VGluT (vesicular glutamate transporter; 1:1000) were then incubated with the fixed proboscis in PBS/0.1\% TX-100/0.1\% BSA overnight at $4^{\circ} \mathrm{C}$ with gentle agitation. Preparations were washed extensively and incubated with secondary antibodies in PBS/0.1\% TX-100/ $0.1 \%$ BSA for $2 \mathrm{~h}$ at room temperature with gentle agitation. After washing, CM9 muscles were dissected from the preparation and mounted on glass slides in SlowFade (Invitrogen) mounting medium, and digital images were captured using a back-cooled Orca digital camera (Hamamatsu) attached to a Zeiss Axiovert immunofluorescent microscope using Slidebook software (Intelligent Imaging Innovations). All Dlg-positive innervations are the result of the single CM9 motor neuron (MN) innervation of the CM9 fibers (Rawson et al., 2012). Dlg-positive innervations were manually counted. For synapse area analysis, all 3D images were randomized and blinded. Surface area of VGluT immunoreactivity was determined using the segmented masking option in Slidebook, which effectively selected VGluT immunoreactivity in the $3 \mathrm{D}$ images based on contrast, and total surface area of VGluT immunoreactivity was determined using Slidebook software.

CM9 NMJ electrophysiology. Dissections and recordings were performed in a modified HL3 solution (containing, in mM: $70 \mathrm{NaCl}, 5 \mathrm{KCl}$, $10 \mathrm{NaHCO}_{3}, 5$ trehalose, 115 sucrose, 5 HEPES, $0.5 \mathrm{CaCl}_{2}, 3 \mathrm{MgCl}_{2}$ ). Flies were suctioned into a Pasteur pipette and placed on top of ice for 15-20 s until the fly lost postural control. The fly was then quickly transferred to a small Sylgard dissection surface where it was decapitated. The head was moved onto its flat posterior surface and the proboscis was then pinned into the extended position, and the entire head was covered in ice-cold dissection solution. The anterior head cuticle containing the antennae was dissected from the preparation. The proboscis was then re-pinned in the retracted position to put tension on the CM9 muscles. A loop of the lateral pharyngeal nerve was drawn into a suction electrode filled with modified HL3 (pulled glass capillary tube with a fire-polished tip, $\sim 15 \mu \mathrm{m}$ opening) and stimulated at $0.5-5 \mathrm{~V}$ for $300 \mu \mathrm{s}$ (Digitimer Ltd., Model DS2A). The presence of a presynaptic action potential-based EPSP was verified by the presence of a distinct voltage threshold for EPSP appearance. Intracellular recordings were made on the most cranial CM9 muscle fiber accessible from the anterior side with a sharp recording electrode $(\sim 30 \mathrm{M} \Omega$, filled with $3 \mathrm{~m}$ potassium chloride). The overall organization of the fibers is highly stereotyped from animal to animal and across age, so it is likely we are interrogating the same fiber in each recording. This fiber has two Dlg-positive innervations. A Neuroprobe Amplifier Model 1600 (A-M Systems) was used in combination with a PowerLab 4/30 (ADInstruments) to amplify and digitize the data. LabChart7 (ADInstruments) was used to record the data and MiniAnalysis (Synaptosoft) was used to measure both miniature EPSP (mEPSP) and EPSP events. Muscle membrane resistance was calculated using the change in muscle potential in response to current injection. Instantaneous resting membrane potential was determined by measuring the initial potential reading when the recording electrode first penetrated the muscle membrane.

For acute pharmacological homeostatic challenge, Philanthotoxin433 (PhTx; Sigma-Aldrich) was used from a stock solution of $4 \mathrm{~mm}$ (in DMSO) and diluted in HL3 saline to a working concentration of $10 \mu \mathrm{M}$ in normal recording saline. For hypertonic stimulation of readily releasable vesicle pools, normal recording saline was initially applied to the preparation to record baseline spontaneous activity before being replaced with recording saline supplemented with sucrose to a total final concentration of $415 \mathrm{~mm}$, and recordings continued for $120 \mathrm{~s}$ in hypertonic saline.

Statistical analysis. All multiple comparisons were performed using a one-way ANOVA with a Bonferroni correction for multiple comparisons. Frequency distributions in response to hypertonic stimulation were compared using both a Kolmogorov-Smirnov test and a Mann-Whitney analysis. All two-way comparisons were performed using a standard Student's $t$ test. All statistical analysis was performed using GraphPad Prism 6 software.

\section{Results}

\section{Quantal content and EPSP amplitude increase abruptly during aging at the $\mathrm{CM} 9 \mathrm{NMJ}$}

The CM9 NMJ is a glutamatergic synapse located on the proboscis of the adult Drosophila and is an ideal system to investigate the effects of age on neurotransmission (Fig. 1A; Rawson et al., 2012). The presynaptic arbor of the CM9 MN ramifies to make 35 distinct synaptic innervations distributed evenly among all 15 muscle fibers that constitute the CM9 muscle (Fig. 1B; Rawson et al., 2012). The CM9 MN is the only source of glutamatergic input into the CM9 muscle and is necessary and sufficient for CM9 muscle contraction (Gordon and Scott, 2009; Rawson et al., 2012). To assay synaptic vesicle (SV) release from the CM9 NMJ, a recording electrode is placed in the CM9 muscle fiber to record the muscle response to the evoked release of neurotransmitter from the nerve terminal. The nerve bundle containing the CM9 motor neuron nerve fiber is directly stimulated using an en passant configuration to generate action potentials (Fig. 1C). Previous studies have suggested that the depolarization of the muscle, measured by the size of the EPSP, represents a set point that the synaptic homeostatic signaling system defends by adjusting the number of synaptic vesicles released during an action potential [quantal content (QC); Paradis et al., 2001; Davis, 2006]. Importantly for our study, the homeostatic response at the NMJ can be quantitated allowing for comparisons of both the set point and the homeostatic signaling system during aging. Our first question was to determine how stable the set point is during aging by determining the size of the EPSP and the mEPSPs from the CM9 NMJ across the lifespan of the fly (Fig. 1C,D). We observed that EPSP amplitudes are stable from 7 to $35 \mathrm{~d}$, at which time we observe an abrupt increase in the amplitudes of the EPSPs from $8.45( \pm 0.36) \mathrm{mV}$ at $35 \mathrm{~d}$ of age to $12.75( \pm 0.54) \mathrm{mV}$ at $42 \mathrm{~d}$, and that this enhanced level of neurotransmission was stably maintained to at least $60 \mathrm{~d}$ of age (Fig. $1 D$; Table 1). We did not observe changes in mEPSP amplitudes over the same time span (Fig. 1D). 

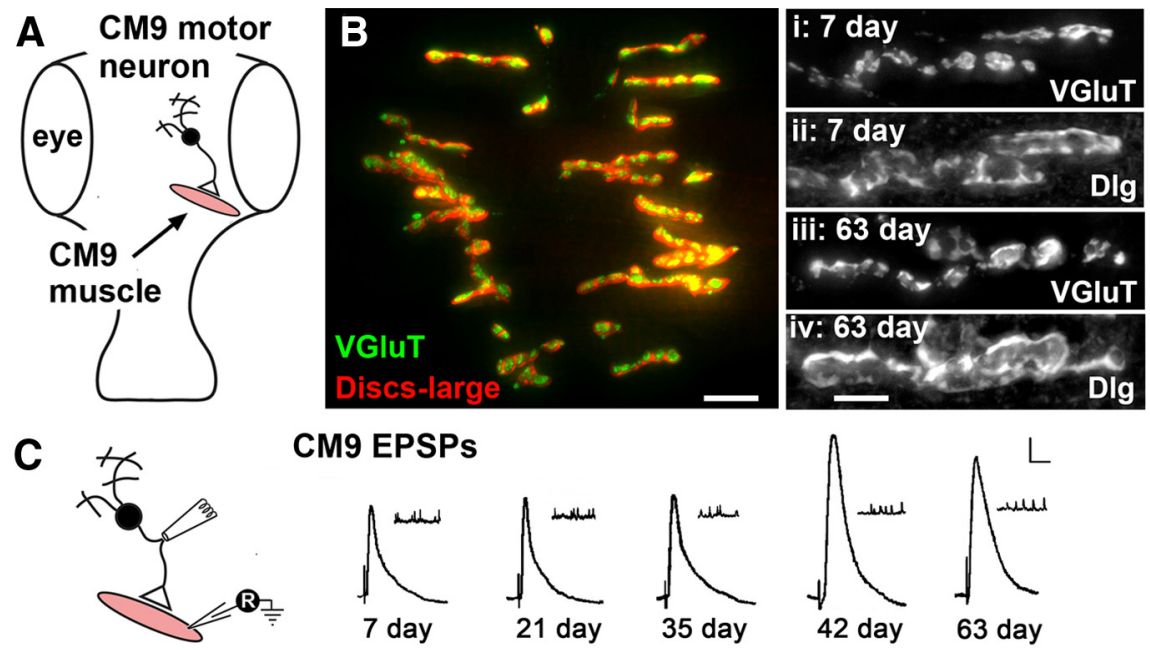

CM9 EPSPs
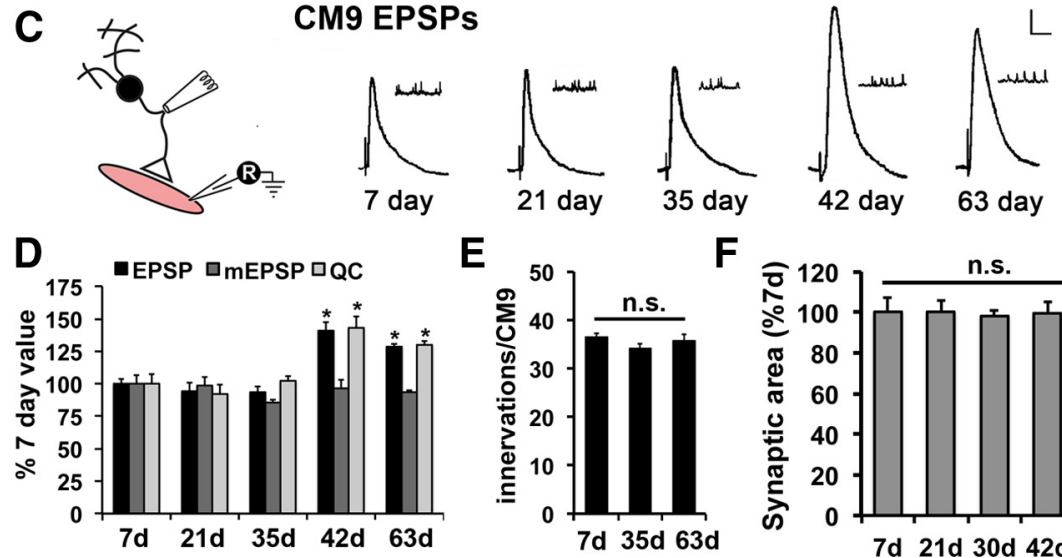

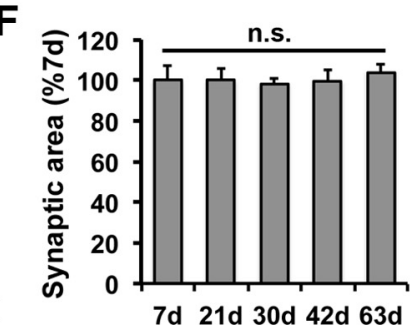

Figure 1. Age-dependent increases in quantal content and the EPSP amplitude. $\boldsymbol{A}$, Diagram of Drosophila head indicating the approximate location of the CM9 muscle and the CM9 MN. $\boldsymbol{B}$, Immunofluorescent image of 7-d-old CM9 NMJs stained with antibodies against the presynaptic VGluT protein and the postsynaptic Discs-large protein. Scale bar, $50 \mu \mathrm{m}$. $\boldsymbol{i}$-iv, Panels show high magnification of VGluT (i, iii) and Dlg (ii, iv) staining from 7-d-old (i, ii) and 63-d-old (iii, iv) NMJs. Scale bar, $5 \mu \mathrm{m}$. $\boldsymbol{C}$, Recording arrangement and representative traces of CM9 EPSP and mEPSPs from flies of indicated ages. Calibration: $2 \mathrm{mV}, 10 \mathrm{~ms}$. $D$, Graph of average values for EPSP (black bars), mEPSP (dark gray bars), and QC (light gray bars) normalized to the $7 \mathrm{~d}$ value for each. ${ }^{*} p<0.01$, significant differences versus 7, 21, and $35 \mathrm{~d}$ values for both QC and EPSP amplitudes. Error bar indicates SEM. $\boldsymbol{E}$, Graph of the average number of distinct Dlg-positive innervations on the CM9 muscles by the CM9 motor neuron determined by immunofluorescence microscopy. Error bars indicate SEM. $\boldsymbol{F}$, Graph of the average surface area $\left(\mu \mathrm{m}^{2}\right)$ of the total VGluT immunoreactivity per CM9 NMJ normalized to 7-d-old values. Error bars indicate SEM.

There were also no significant changes in the input resistance of the CM9 muscle or the resting membrane potential of the muscles during this time span (Table 1). These data suggested that the increase in EPSP observed with age was due to an increase in the number of synaptic vesicles released during activity. In support of this suggestion, we found significant increases in quantal content with age, determined by dividing the EPSP by the mEPSP for each synapse, which mirrored the abrupt increase in EPSP amplitudes observed between 35 and $42 \mathrm{~d}$ of age (Fig. 1D; Table 1). In addition, this enhanced level of QC was maintained to at least $63 \mathrm{~d}$ of age (Fig. 1D). Note that under the conditions and diet used in these studies, our median lifespan for control flies is $45 \mathrm{~d}$ of age, and $63 \mathrm{~d}$ of age is near the 85th percentile of lifespan (Rawson et al., 2012).

We wondered whether this change in QC might be the result of a change in the size of the innervation of the CM9 muscle by the CM9 MN. To investigate this possibility, we performed immunofluorescence microscopy on CM9 NMJs of various ages using antibodies to the presynaptic VGluT and the postsynaptic PSD-95 homolog Dlg (Fig. 1B). This staining allows us to easily analyze both the innervation pattern and synaptic substructures of the CM9 NMJs during aging (Fig. $1 B i-i v$ ). At the level of innervation, we observe no change in the average number of synaptic contacts made upon the CM9 muscle with increasing age (Fig. 1E; Rawson et al., 2012). Using segmentation analysis of pixel intensities for VGluT fluorescence from deconvolved 3D images, we quantified the total surface area of the VGluT staining at CM9 NMJs as a function of age and found no significant change in total synaptic area during aging (Fig. $1 F$ ). We also see no obvious changes in the subsynaptic staining patterns of VGluT or Dlg (Fig. $1 B i-i v$ ). Together, these data support the idea that both the EPSP amplitude and quantal content experience a stable increase during aging that is not due to increased synaptic innervation.

\section{The increase in quantal content at $42 \mathrm{~d}$ of age is not blocked by ephexin mutations}

It is possible that the change in EPSP and QC at $42 \mathrm{~d}$ of age does not represent a change in the set point but rather is a homeostatic response to the declining motor function observed during this time period (data not shown). To investigate this possibility, we investigated whether ephexin mutant synapses also experienced the agedependent increase in EPSP amplitudes and QC. The ephexin gene encodes a Drosophila Eph receptor that has previously been shown to be required within the larval nerve terminal in a chronic model of synaptic homeostasis (Petersen et al., 1997; Frank et al., 2009). In ephexin mutants, we observe a robust increase in both EPSP amplitudes and QC (Fig. 2A, C; Table 1 ) that is similar to wild-type controls analyzed in parallel under identical conditions (Fig. 2B). These results are consistent with the interpretation that the increases in EPSP amplitude and QC observed with age are not a homeostatic response. Note that, similar to early studies at the larval NMJ, we find that ephexin mutants have a significant reduction in QC and EPSP amplitude (Table 1; Frank et al., 2009). Thus, Ephexin plays an essential role during basal neurotransmission at the CM9 NMJ. It is interesting that despite a role for Ephexin during normal neurotransmission, we see a robust increase in QC and EPSP amplitudes between 7 and $42 \mathrm{~d}$ of age in ephexin mutants (Fig. 2C).

\section{Pharmacologic induction of synaptic homeostasis at the 7-d-old CM9 NMJ}

Application of PhTx, a blocker of insect ionotropic glutamate receptors, to the Drosophila larval NMJ has previously been used to initiate a quantitative homeostatic response (Frank et al., 2006). Briefly, application of sub-blocking concentrations of PhTx to larval NMJs for 30 s reduces the amplitudes of both the mEPSPs and EPSP. Continued incubation in the presence of PhTx finds that after a period of $\sim 10 \mathrm{~min}$, the EPSP amplitudes return to near pretoxin levels, whereas the amplitudes of the mEPSPs remain reduced. The increase in the EPSP amplitudes is accomplished by an increase in the number of synaptic vesicles fusing during an action potential (quantal content; Frank et al., 2006). In the present study, we will consider the number of quanta released as a measure of the magnitude of the homeostatic response and the amplitude of the EPSP after prolonged treatment 
Table 1. Analysis of quantal release with increasing age at the CM9 NMJ

\begin{tabular}{|c|c|c|c|c|c|c|c|}
\hline Genotype & Age (days) & EPSP amplitude & mEPSP amplitude & Quantal Content & $\mathrm{RMP}(\mathrm{mV})$ & $\mathbb{I R}(\mathrm{M} \Omega)$ & $n$ \\
\hline \multirow[t]{5}{*}{$w^{1118}$} & 7 & $9.03 \pm 0.33$ & $1.12 \pm 0.08$ & $8.55 \pm 0.61$ & $-37.43 \pm 1.44$ & $8.43 \pm 0.81$ & 13 \\
\hline & 21 & $8.48 \pm 0.62$ & $1.11 \pm 0.62$ & $7.90 \pm 0.07$ & $-43.05 \pm 1.70$ & $7.83 \pm 1.33$ & 14 \\
\hline & 35 & $8.45 \pm 0.36$ & $0.97 \pm 0.02$ & $8.75 \pm 0.32$ & $-41.91 \pm 1.02$ & $8.50 \pm 1.01$ & 10 \\
\hline & 42 & $12.75 \pm 0.55$ & $1.13 \pm 1.43$ & $12.22 \pm 0.75$ & $-43.53 \pm 2.16$ & $8.88 \pm 0.88$ & 16 \\
\hline & 63 & $11.63 \pm 0.18^{a}$ & $1.05 \pm 0.03$ & $11.11 \pm 0.26^{a}$ & $-46.96 \pm 2.82$ & $8.60 \pm 0.99$ & 10 \\
\hline \multirow[t]{2}{*}{ ephexin } & 7 & $5.70 \pm 0.36^{b}$ & $1.02 \pm 0.03$ & $5.57 \pm 0.36^{b}$ & $-42.69 \pm 1.65$ & $9.07 \pm 1.85$ & 7 \\
\hline & 42 & $11.30 \pm 0.20$ & $1.10 \pm 0.05$ & $10.38 \pm 0.39^{b}$ & $-57.56 \pm 1.44$ & $8.00 \pm 0.82$ & 8 \\
\hline \multirow[t]{2}{*}{$w^{1118}$} & 7 & $9.43 \pm 0.33$ & $1.08 \pm 0.08$ & $9.25 \pm 0.71$ & $-39.15 \pm 1.23$ & $8.50 \pm 1.44$ & 13 \\
\hline & 42 & $12.17 \pm 0.49$ & $1.02 \pm 0.02$ & $12.05 \pm 0.53$ & $-53.59 \pm 1.37$ & $8.22 \pm 1.19$ & 13 \\
\hline
\end{tabular}

All recordings were performed under identical conditions and all values are presented as averages \pm SEM. Datasets for each genotype and condition consist of at least 2 separate data collection sessions. Value for $n$ represents the number of recordings. Only one recording is performed per animal. Units for EPSP and mEPSP are mV. RMP, Resting membrane potential; IR, depolarizing input resistance of CM9 muscle. Within data columns, all values that are significantly different from $7 \mathrm{~d}$ value are in bold as determined using a one-way ANOVA with a Bonferroni post-hoc test for multiple comparisons $(p<0.01)$ or Student's $t$ test for pairwise comparison ( $p<0.01$ ).

${ }^{a}$ There is no significant difference in the values for EPSP and QC between the 42- and 63-d-old NMJs.

${ }^{b}$ These values from ephexin mutants are significantly different from the values for their $w^{1118}$ controls as determined from pairwise comparison using a Student's $t$ test $(p<0.01)$.

with PhTx a measure of the precision of the homeostatic response. Importantly, both of these aspects of synaptic homeostasis are highly quantitative, allowing for robust statistical analyses.

Using a similar pharmacological approach to induce homeostasis, we have investigated the synaptic homeostatic response at the CM9 NMJ. Addition of 10 $\mu \mathrm{M}$ PhTx to the CM9 NMJ induced a rapid and significant reduction in mEPSP and EPSP amplitudes within $30 \mathrm{~s}$ of addition of PhTx (Fig. 3B-E; Table 2). Although the effects of PhTx on mEPSP amplitudes were consistent throughout the recording period (Fig. $3 B, E$ ), we noticed that the amplitudes of the EPSPs continued to decline until they reached a nadir at $\sim 5 \mathrm{~min}$, after which the EPSP amplitudes began a gradual return to pretoxin levels, which required $\sim 20 \mathrm{~min}$ (Fig. $3 A, C, D)$. The return of EPSP amplitudes to near pretoxin levels at $20 \mathrm{~min}$ is accomplished by a large increase in the QC of the release event at the CM9 NMJ consistent with a robust synaptic homeostatic response at 7-d-old CM9 NMJs (Fig. 3E; Table 2). This homeostatic profile is similar to what has been reported at the larval NMJ, although the timescale is longer at the CM9 NMJ compared with what has been reported at the larval NMJ, which has been shown to achieve homeostatic compensation within 10 min of PhTx application (Frank et al., 2006). In addition, the further reduction in EPSP amplitudes and QC observed at 5 min has not been reported at the larval NMJ. Previous studies in the larvae did not report EPSP amplitudes or QC between 30 and $200 \mathrm{~s}$ of PhTx incubation, so it is possible that the temporary decline in EPSP amplitudes and QC that we observe at 5 min could have been missed in the previous analysis (Frank et al., 2006). We do not currently know the mechanisms underlying this reduction in QC at $5 \mathrm{~min}$, but because we see a robust increase in QC at 20 min, we do not believe that this reflects an effect of PhTx on presynaptic release mechanisms, per se, but rather reflect either the machinations of the presynaptic homeostatic response or an overestimation of the mEPSP amplitudes due to an increase in release events that are below our detection limit. Regardless, the homeostatic response returns the EPSP amplitudes to near pretoxin levels consistent with a fully functioning homeostatic response. Finally, the apparent reduction in mEPSP frequency in the presence of PhTx has also been observed at the
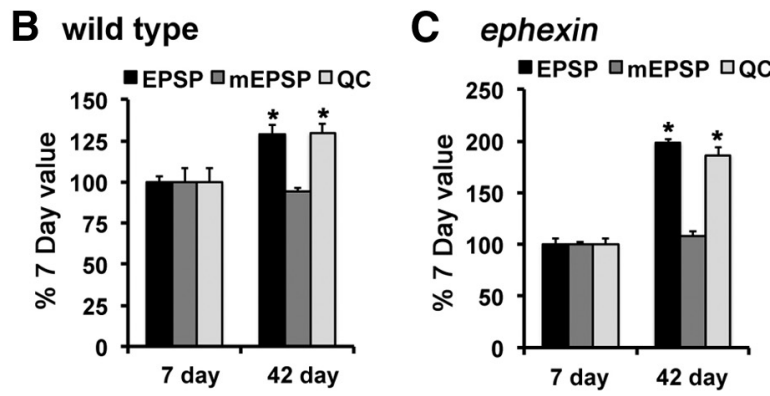

Figure 2. Age-dependent increases in quantal content and the EPSP amplitude exist in ephexin mutants. $\boldsymbol{A}$, Representative tion: $2 \mathrm{mV}, 10 \mathrm{~ms}, \boldsymbol{B}$ Graphs of EPSP amplitude (black bars), mEPSP (dark gray bars), and QC (light gray bars) for wild-type control EPSP amplitude (black bars), mEPSP (dark gray bars), and QC (light gray bars) for ephexin mutant NMJs. For both QC and EPSP, amplitude is significantly increased at 42-d-old NMJs compared with 7-d-old NMJs.

larval NMJ and has been hypothesized to be either the result of the failure to detect all spontaneous events in the presence of PhTx, or a sign that mEPSP frequency is not indicative of presynaptic function at these synapses (Frank et al., 2006).

To further characterize this homeostatic response at the CM9 $\mathrm{NMJ}$, we investigated the requirement for the ephexin gene during the homeostatic response to the application of PhTx. We find that application of PhTx to CM9 NMJs in 7-d-old ephexin mutants initially reduces the amplitudes of both EPSPs and the mEPSPs by $\sim 50 \%$, similar to what was observed at wild-type CM9 NMJs (Fig. 3C,F,G; Table 2). In contrast to what we observed in wild-type flies, we found that after 20 min of incubation in PhTx, the amplitudes of the EPSPs and the size of the QC in ephexin mutants were unchanged from the 5 min values, demonstrating a suppression of the homeostatic response by the presence of the ephexin mutation (Fig. 3C,F,G). These data demonstrate that the homeostatic response to the application of PhTx to the CM9 NMJ requires the activity of the ephexin gene product, an Eph receptor homolog. Combined with our previous data demonstrating an increase in the EPSP amplitude during aging in ephexin mutants, these data further support the notion that the change in the EPSP and QC at $42 \mathrm{~d}$ of age is not a homeostatic response.

\section{The synaptic homeostatic response is increased at the} 42-d-old NMJ

We reasoned that if the change in the EPSP amplitude and QC observed at $42 \mathrm{~d}$ was due to an ongoing homeostatic response, the response to experimentally induced homeostasis at 42 -d-old NMJs might be occluded. Therefore, we investigated the homeo- 


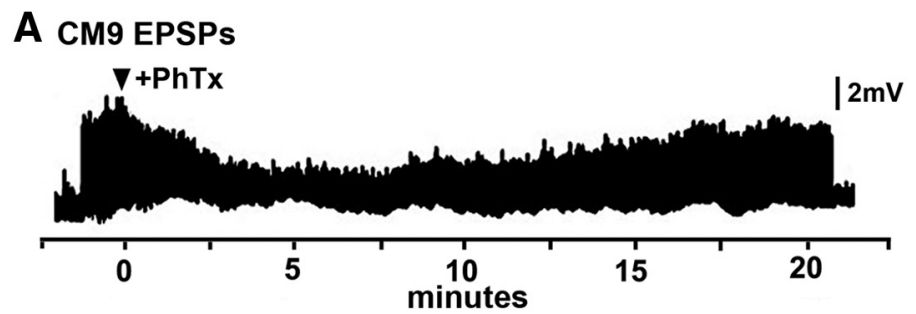

\section{B 7 day-old mEPSPs}

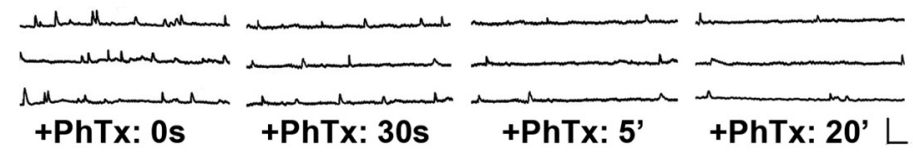

\section{7 day-old EPSPs}

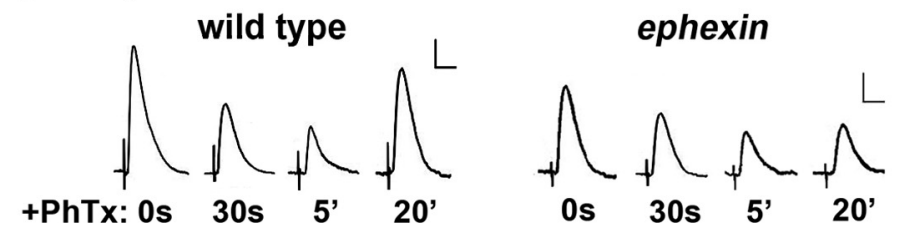

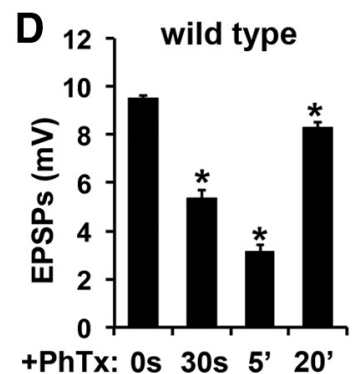
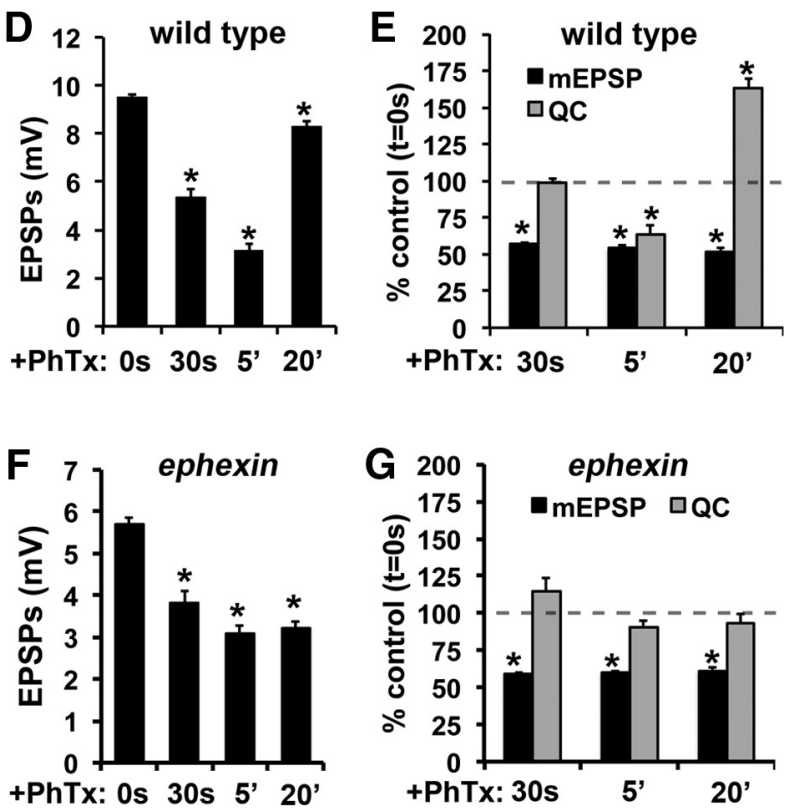

Figure 3. The homeostatic response to Philanthotoxin application to the 7-d-old CM9 NMJ. $A$, Representative trace of EPSP amplitudes during the real-time homeostatic response of CM9 NMJs to the application of $10 \mu \mathrm{M}$ PhTx. EPSPs evoked at $1 \mathrm{~Hz}$. B, Representative mEPSP traces from wild-type 7-d-old CM9 NMJs. Application of PhTx to the NMJ reduces the amplitude of the mEPSP compared with the value before PhTx application $(0 \mathrm{~s})$ due to the blockage of the postsynaptic glutamate receptors in response to the toxin. Calibration: $2 \mathrm{mV}, 10 \mathrm{~ms}$. C, Representative traces of evoked EPSPs from a wild-type and an ephexin mutant NMJ treated with PhTx for 0 (value immediately before application of PhTx), 30 s, 5', and 20'. Calibration: $2 \mathrm{mV}, 10 \mathrm{~ms}$. D, Graphs of the average EPSP amplitude in wild-type flies treated with PhTx for $0 \mathrm{~s}, 30 \mathrm{~s}, 5^{\prime}$, and $20^{\prime}$. All EPSP amplitudes are significantly different from the value for the $t=0 \mathrm{~s}$ time point $\left({ }^{*} p<0.01\right.$; ANOVA). Values for EPSP amplitudes at $30 \mathrm{~s}, 5^{\prime}$, and $20^{\prime}$ are significantly different from each other $(p<0.01$; ANOVA). $E$, Graphs of $\mathrm{mEPSP}$ and QC values in wild-type flies in response to the application of PhTx for $30 \mathrm{~s}$, $5^{\prime}$, and $20^{\prime}$. All values are normalized to the $t=0^{\prime}$ value. These values for mEPSP amplitudes and $Q C$ are significantly different compared with $t=00^{\prime}$ value $\left({ }^{*} p<0.01 ;\right.$ ANOVA). $F_{,}$Graphs of the average EPSP amplitude in ephexin mutant flies treated with PhTx for $30 \mathrm{~s}, 5^{\prime}$, and $20^{\prime}$. EPSP amplitudes are significantly different from the value for the $t=0^{\prime}$ value $\left({ }^{*} p<0.01\right.$; ANOVA). G, Graphs of $\mathrm{mEPSP}$ and $\mathrm{QC}$ values in ephexin mutant in response to the application of PhTx. All values are normalized to the $t=0^{\prime}$ value. Note that the increase in $Q^{\prime}$ observed between $5^{\prime}$ and $20^{\prime}$ in wild-type flies is absent in ephexin mutant NMJs. These values are significantly different compared with $t=0$ min value $\left({ }^{*} p<0.01\right.$; ANOVA).

Table 2. Electrophysiological values for acute homeostasis analysis at the CM9 NMJ

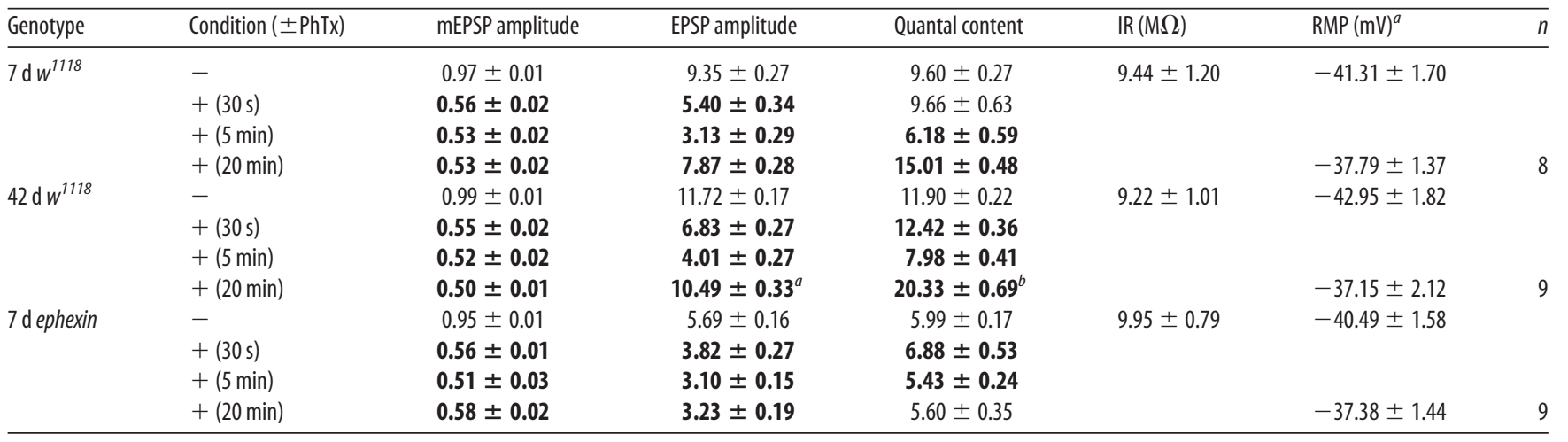

All recordings were performed under identical conditions and all values are presented as averages \pm SEM. Datasets for each genotype and condition consist of at least 2 separate data collection sessions. Value for $n$ represents the number of recordings. Only one recording is performed per animal. RMP, resting membrane potential; IR, depolarizing input resistance of $\mathrm{CM} 9 \mathrm{muscle}$. The $(-\mathrm{PhTx})$ value represents the value prior to addition of $\mathrm{PhTx}\left(t=0^{\prime}\right)$. Within the $\mathrm{mEPSP}$ EPSP, and quantal content columns and within the three genotypes, all bold values are significantly different from their respective - PhTx value as determined using a one-way ANOVA with a Bonferroni post-hoc test for multiple comparisons $(p<0.01)$.

${ }^{a}$ There are no significant differences in any genotype between the ( $-\mathrm{PhTx}$ ) and the (+PhTx, 20 min) values for RMP (Student's t test).

${ }^{b}$ The values for EPSP and QC after 20 min of PhTx incubation at $42 \mathrm{~d}$ is significantly different from the value at $7 \mathrm{~d}(p<0.01$, Student's $t$ test).

static response at the 42 -d-old NMJ to compare it to the response we observed at the 7-d-old NMJ. We observed that incubation with PhTx of CM9 NMJs in 42-d-old animals for 30 s resulted in a similar reduction in $\mathrm{mEPSP}$ and EPSP amplitudes compared with what was observed at 7-d-old CM9 NMJs, demonstrating that the sensitivity of the postsynaptic receptors to PhTx does not change between 7 and $42 \mathrm{~d}$ of age (Fig. 4A,D; Table 2). There are also no differences in the resting membrane potential or input resistance after application of $\mathrm{PhTx}$ between 7 - and 42-d-old NMJs (Table 2). These data support the idea that the effects of PhTx on synapse function are very similar at 7-d-old and 42-d- old NMJs. Continued incubation in PhTx for 20 min resulted in a return of the EPSP amplitude to near the pretoxin level (Fig. $4 B-D)$. This rebound in EPSP amplitude was the result of a large increase in QC consistent with a robust homeostatic response demonstrating that homeostatic plasticity is not occluded at the 42-d-old NMJ (Fig. 4D; Table 2).

Importantly, the final EPSP amplitudes established by the 42d-old NMJ after incubation with PhTx for $20 \mathrm{~min}$ are significantly larger than the EPSP amplitudes established by the homeostatic response at the 7-d-old NMJ (Fig. 4E). Analysis of the QC of the homeostatic responses at 7 and $42 \mathrm{~d}$ reveals that the 
difference in the amplitudes of the EPSPs generated after incubation with $\mathrm{PhTx}$ for $20 \mathrm{~min}$ is due to a significant increase in the QC of the homeostatic response at the 42-d-old NMJ compared with the QC of the response at the 7-d-old NMJ (Fig. 4F, $p<0.01)$. These analyses support the finding that the homeostatic response observed at the 42-d-old synapse is actually larger than the homeostatic response observed at the 7 - $\mathrm{d}$-old synapse. This is supported by analysis of the magnitude of the change in EPSP amplitudes and QC between 5 and $20 \mathrm{~min}$ of PhTx incubation, which are also significantly increased at 42-d-old NMJs compared with 7-d-old NMJs (Fig. 4G,H). Because the increased homeostatic response observed at 42-dold NMJs is appropriate for the larger EPSP amplitudes of the 42-d-old NMJ, these data support a change in the homeostatic set point of the CM9 NMJ between 7 and $42 \mathrm{~d}$ of age.

\section{The 42-d-old NMJs display enhanced synaptic depression}

Under normal conditions, the rate of exocytosis is balanced by a rate of endocytosis, which ensures that the supply of releasable synaptic vesicles is maintained during activity. Situations that perturb this balance, such as reduced endocytosis or enhanced SV release, can lead to depression of EPSP amplitudes during trains of action potentials due to the reduction in the number of available SVs. We wondered whether the increase in release that accompanies our change in set point left the synapse susceptible to synaptic depression during trains of action potentials or whether the change in the set point includes enhanced endocytosis. To investigate this possibility, EPSP amplitudes were monitored during trains of action potentials at 7-d-old and 42-d-old NMJs (Fig. 5). Values for the initial EPSP amplitude (initial), the EPSP amplitude at $7.5 \mathrm{~s}(1 / 2)$, and the $15 \mathrm{~s}$ EPSP amplitude (final) were generated for each animal by averaging the nearest 3 EPSPs for each time point. We observed a robust and significant depression of EPSP amplitudes at $7.5 \mathrm{~s}$ (Fig. 5B, right, $1 / 2 ; C$ ) and at $15 \mathrm{~s}$ (Fig. 5B, right, final; $C$ ) at 42 -d-old NMJs subjected to stimulation at $40 \mathrm{~Hz}$. This synaptic depression was not observed at 7-d-old NMJs subjected to the same stimulation protocol (Fig. $5 B$, left; $C$ ). The synaptic depression we observed was also dependent upon stimulation frequency since stimulation at $20 \mathrm{~Hz}$ did not result in a significant change in EPSP amplitudes at 42-d-old NMJs (Fig. $5 C ; p=0.04$, final value vs. initial using Student's $t$ test). Note that these stimulation frequencies are in line with endogenous firing rates at Drosophila motor neurons (Chouhan et al., 2010). These data support the theory that the potentiation of SV release accompanying the change in the homeostatic set point results in enhanced susceptibility of the 42-d-old NMJ to synaptic depression.

We also investigated whether the change in SV release observed with age was the result of an increase in the size of the readily releasable pool (RRP) of synaptic vesicles. For this analysis we used a hyperosmotic stimulus approach, which has been used
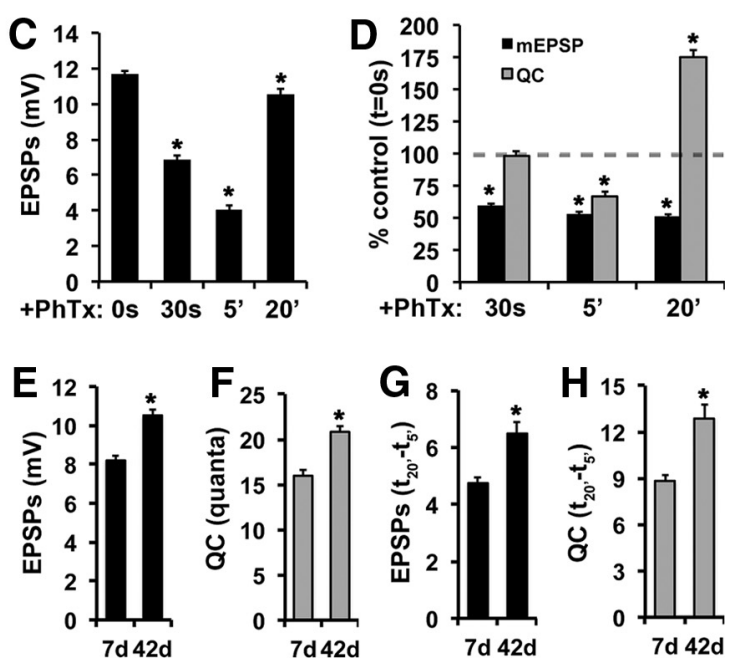

Figure 4. Analysis of the homeostatic response to Philanthotoxin application at 42-d-old CM9 NMJs. $A$, Representative mEPSP

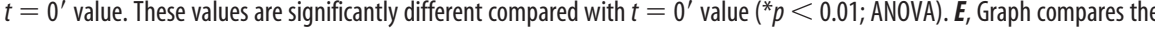
QC from the $20^{\prime}$ value of QC for each animal. The magnitude of the release event during homeostasis at the 42-d-old NMJ is significantly larger than the magnitude of the release event during homeostasis at the 7-d-old NMJ ( ${ }^{*} p<0.001$; Student's $t$ test).

extensively to estimate the sizes of the RRP at numerous synapses including the Drosophila larval NMJ (Rosenmund and Stevens, 1996; Stevens and Williams, 2007; Müller and Davis, 2012). Consistent with previous studies, application of a hypertonic recording saline to either 7-d-old NMJs or 42-d-old CM9 NMJs elicited a rapid increase in the frequency of spontaneous release events (Fig. 6A). Quantification of these frequencies in $10 \mathrm{~s}$ bins over the period of the experiment ( $120 \mathrm{~s}$ ) finds no significant change in the distribution of the average frequencies of mEPSPs between 7-dold and 42-d-old NMJs (Fig. 6B). There is also no significant difference in the average bin frequencies (Fig. $6 B$ ) or in the total number of quanta released during the hypertonic stimulation between 7-d-old and 42-d-old NMJs (Fig. 6C). These data demonstrate that the increase in QC observed with age is not accompanied by an increase in the size of the RRP.

\section{Discussion}

A change in the set point for synaptic homeostasis during aging

Previous studies at the larval NMJ have established that the depolarization of the muscle, measured by the amplitude of the EPSP, represents a homeostatic set point that is defended by precise changes in SV release (Frank, 2013). We have found that the amplitude of the EPSP at the adult CM9 NMJ is very stable between 7 and $35 \mathrm{~d}$ of age, consistent with the EPSP representing a set point that is being actively maintained by a homeostatic signaling system. Consistent with this interpretation, challenging 
A
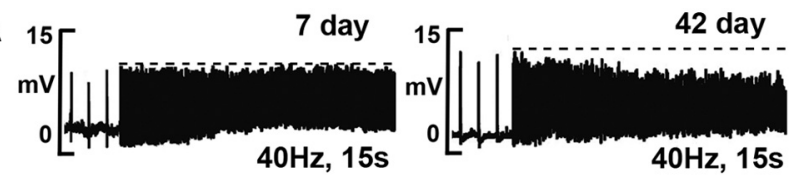

B
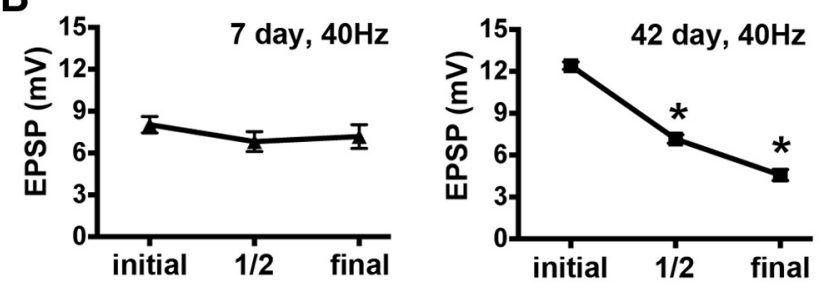

C

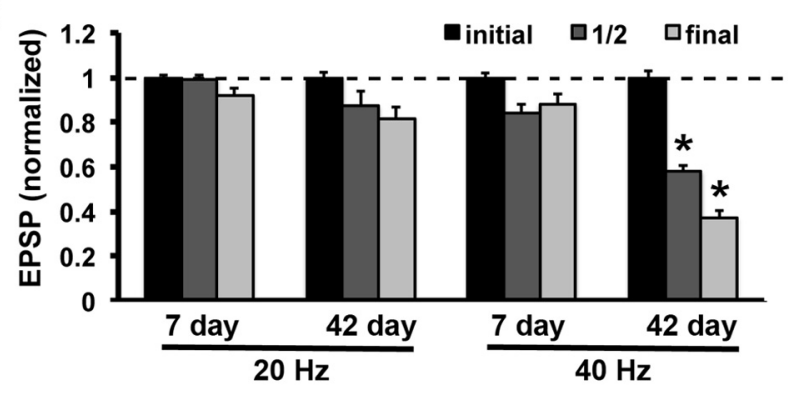

Figure 5. Synaptic depression at the 42-d-old CM9 NMJ. $\boldsymbol{A}$, Representative traces of EPSPS from a 7-d-old CM9 NMJ or 42-d-old CM9 NMJ during a $15 \mathrm{~s}$ train of action potentials at $40 \mathrm{~Hz}$. $B$, The CM9 motor neuron was stimulated at $40 \mathrm{~Hz}$ for $15 \mathrm{~s}$. The average values ( $n=8$ animals) for EPSPs corresponding to the initial amplitudes (initial), the amplitude of the EPSPs after $7.5 \mathrm{~s}$ $(1 / 2)$, and the final EPSP amplitude (final) produced during the $15 \mathrm{~s}$ stimulation. Note that the only condition that showed significant synaptic depression was the 42-d-old NMJ subjected to $40 \mathrm{~Hz}$ stimulation ( ${ }^{*} p<0.001$, ANOVA; error bars indicate SEM). C, Graphs represent the EPSP values from CM9 NMJs stimulated at either 20 or $40 \mathrm{~Hz}$. For each condition, the values are normalized to the value of the initial EPSP. ${ }^{*} p<0.001$ versus initial value and each other (ANOVA). Error bars indicate SEM. $n=8$ animals for each condition.

A

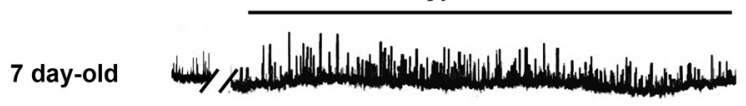

42 day-old time:

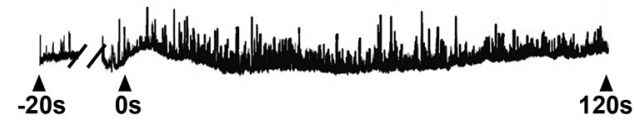

B

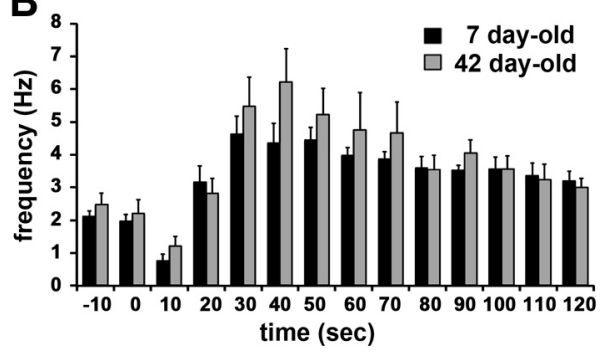

C

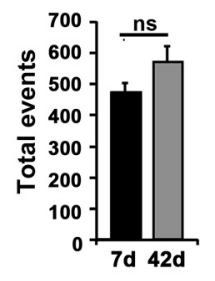

Figure 6. The size of the sucrose-sensitive synaptic vesicle pool does not change at $42 \mathrm{~d}$ of age. $\boldsymbol{A}$, Representative traces of mEPSP recordings during the incubation (120 s) of the NMJ in hyperosmotic recording saline $(420 \mathrm{~mm})$. $\boldsymbol{B}$, Histogram of average mEPSP frequency between $7 \mathrm{~d}$ (black bars) and $42 \mathrm{~d}$ (gray bars) NMJs over the entire 120 s incubation presented in 10 s bins. There are no significant differences in the distributions or peak values. $C$, The average number of total quanta released during an osmotic shock at $42 \mathrm{~d}$ (gray bars) is not significantly different from the total quanta released at $7 \mathrm{~d}$ (black bars). these young synapses with the glutamate receptor antagonist PhTx, which reduces mEPSP and EPSP amplitudes, shows that the EPSP amplitude rapidly and precisely returns to pretoxin levels (Fig. 3). These results are consistent with the existence of a homeostatic signaling system at the CM9 NMJ that is similar to the homeostatic system previously defined at the larval NMJ. Further analysis finds that the EPSP amplitude at the CM9 NMJ abruptly increases to an enhanced level at $42 \mathrm{~d}$ of age that is then stably maintained at this enhanced level as the animal continues to age (Fig. 1). We have observed this stable increase in EPSP amplitude at $42 \mathrm{~d}$ of age in all animals analyzed, consistent with this age-dependent event representing a change in the baseline function of the synapse. Further, the observation that this enhanced EPSP amplitude is stably maintained suggests that this enhanced EPSP amplitude is being actively maintained by the synaptic homeostatic signaling system. Consistent with this interpretation, we find that challenging of the 42-d-old NMJ with PhTx results in a homeostatic response that returns the EPSP amplitude to the 42-d-old level (Fig. 4). We observe that the values for both EPSP amplitudes and QC after PhTx application are significantly larger at 42-d-old NMJs compared with 7-d-old NMJs (Fig. 4E,F). We also observe that the magnitude of the change in EPSP amplitude and QC, defined as the difference between the values at $5 \mathrm{~min}$ of PhTx incubation and the values after $20 \mathrm{~min}$ of PhTx incubation, are significantly larger at 42-dold NMJs compared with 7-d-old NMJs (Fig. 4G,H). These data support the finding that the synaptic homeostatic signaling system is actively maintaining this enhanced EPSP amplitude and suggests that the change in EPSP amplitude observed at $42 \mathrm{~d}$ of age represents a stable change in synapse function that is defended by homeostatic signaling.

It is interesting that despite the difference in the magnitude of the homeostatic response, the percentage change in QC is nearly identical between the 7- and 42-d-old NMJs. One interpretation of this result is that the response of the CM9 NMJ to PhTx application is not homeostatic, per se, but rather reflects an inherent compensatory mechanism that simply doubles SV release in response to the 50\% decrease in mEPSP amplitudes, independent of the EPSP (i.e., the set point). This possibility is supported by observations from the larval NMJ that the response to PhTx application does not require the occurrence of an EPSP and that only the presence of reduced mEPSP amplitudes is sufficient to trigger the response (Frank et al., 2006). These two mechanisms, compensation versus homeostasis, are difficult to distinguish between, from our dataset. For example, the goal of a homeostatic signaling system would be to return the EPSP amplitude to pretoxin levels, which would require a $200 \%$ increase in QC to offset the $50 \%$ reduction in mEPSP amplitudes. In addition, both mechanisms would need to use a retrograde signal that would alter presynaptic release in response to the application of PhTx. The difference in these mechanisms is that homeostatic signaling systems use feedback control mechanisms in which "sensors" in the muscle detect perturbations to the system and initiate homeostatic effectors that drive the compensatory response. Because it is currently unclear how the muscle senses and responds to changes in its sensitivity to neurotransmitter, it is impossible to distinguish between these two models. Although our data cannot rule out a compensatory mechanism that simply responds to reductions in mEPSP amplitudes, the stability of the increased EPSP in older animals and the precise return to that level after challenge with PhTx is consistent with the increased EPSP amplitude at 42-d-old NMJs, representing a new set point for synaptic homeostasis. 
Currently, we do not know the mechanisms responsible for the increase in neurotransmission observed at $42 \mathrm{~d}$ of age, but the changes in quantal content observed with age reflect a change in the probability SV release. Since the change in QC at $42 \mathrm{~d}$ of age does not involve an increase in synaptic innervation or an increase in the size of the readily releasable pool of SVs, we assume that the change in SV release reflects a change in the mechanisms involved in SV exocytosis. This could involve increased calcium entry during the action potential, a change in the calcium-sensor, or changes in machinery controlling vesicle exocytosis. In support of a change in calcium entry during activity, age-dependent changes in the activity of voltage-gated calcium channels have been well documented throughout the nervous system and could represent a fundamental mechanism that alters neurotransmission with age (Campbell et al., 1996; Thibault and Landfield, 1996; Veng et al., 2003; Bissig et al., 2013).

Although this is the first formal demonstration of an agedependent change in the homeostatic set point at any synapse, age-dependent changes in synaptic output have previously been observed at both mammalian NMJs and central synapses, suggesting that age-dependent potentiation of SV release could be a common effect of age at many synapses (Kelly, 1978; Banker et al., 1983; Foster et al., 1991; Robbins, 1992; Dumas and Foster, 1995). We have now shown that the age-dependent change in synapse strength observed at the fly NMJ is not a homeostatic response since the same age-dependent potentiation occurs in ephexin mutants, which we show blocks synaptic homeostasis at the CM9 NMJ. This indicates a clear molecular distinction between the mechanisms regulating the change in presynaptic output during aging and those involved in synaptic homeostasis. This arrangement might be expected of a homeostatic regulatory system in which the set point needs to be adjustable to maintain normal function despite changes in the physiological environment, such as those that occur during aging or disease.

\section{The role for an adjustable set point at the synapse}

It is interesting that we see increased synaptic depression at $42-$ d-old NMJs compared with 7-d-old NMJs, suggesting that the increase in SV release observed at $42 \mathrm{~d}$ of age results in the depletion of available SVs during high bouts of activity. This suggests that the change in release observed with age is not accompanied by an increase in the endocytosis and replenishment of SV pools. A similar scenario has been reported at mammalian NMJs, where previous studies have demonstrated that not only does presynaptic release increase at some NMJs with age, but that these same nerve terminals exhibit a morphological decrease in the size of the SV pools near the active zone, supporting the notion that the increased SV release creates an imbalance of exocytosis and endocytosis (Banker et al., 1983). Similar findings have also been reported at central synapses (Applegate and Landfield, 1988). At the NMJ, it is possible that if EPSP amplitudes fall too low during activity, muscle contraction could be affected. This possibility leads to the question of whether the increase in the synaptic homeostatic set point is a beneficial event for this system, as might be expected for a homeostatic system with an adjustable set point such as thermoregulation (Cabanac, 2006). Currently, we do not know what is triggering the change in the function of the CM9 NMJ with age, but we suspect that it could be linked to motor function. Like the situation in mammals, Drosophila also exhibit declining motor function with increasing age including the extension of their proboscis, a simple motor reflex that requires CM9 NMJ function (T.K. and B.A.E., data not shown). It is possible that without the increase in EPSP and QC seen at $42 \mathrm{~d}$ of age, the decline in motor function might be more pronounced. This would support the idea that the change in SV release with age is a regulated event designed to preserve motor function and not simply an age-related pathology.

A long-standing question is how positive feedback plasticity (i.e., Hebbian-like) and negative feedback plasticity (i.e., homeostatic) coexist at the synapse to allow the long-lasting changes in synapse strength that are required for normal neural circuit function (Rabinowitch and Segev, 2008; Vitureira et al., 2012; Davis, 2013). One idea is that since homeostatic scaling of synaptic strengths is cell-wide, the relative differences between synapses would be preserved, but findings that homeostatic plasticity can be synapse-specific suggest that this model is too simplistic (Thiagarajan et al., 2005; Branco et al., 2008; Beïque et al., 2011). Another model that incorporates synapse-specific homeostatic plasticity is that synapses undergoing Hebbian plasticity are refractory to global homeostatic mechanisms, allowing cell-wide homeostatic changes in synapse strength while preserving synaptic plasticity (Rabinowitch and Segev, 2008). It is currently unclear how generalizable this computational model is for all neural circuits. Another possibility based on our data is that synaptic homeostatic set points at individual synapses are adjustable, so that the homeostatic regulation of synapse strength can support a long-term change in synaptic strength, such as LTP or the agedependent potentiation observed here (Thiagarajan et al., 2007; Turrigiano, 2012). This creates a scenario in which synapsespecific plasticity, both Hebbian and homeostatic, could coordinate at the single synapse to maintain the stability and plasticity of the individual synapse throughout the lifetime of the organism.

\section{References}

Applegate MD, Landfield PW (1988) Synaptic vesicle redistribution during hippocampal frequency potentiation and depression in young and aged rats. J Neurosci 8:1096-1111. Medline

Banker BQ, Kelly SS, Robbins N (1983) Neuromuscular transmission and correlative morphology in young and old mice. J Physiol 339:355-377. Medline

Beïque JC, Na Y, Kuhl D, Worley PF, Huganir RL (2011) Arc-dependent synapse-specific homeostatic plasticity. Proc Natl Acad Sci U S A 108: 816-821. CrossRef Medline

Bissig D, Goebel D, Berkowitz BA (2013) Diminished vision in healthy aging is associated with increased retinal L-type voltage gated calcium channel ion influx. PLoS One 8:e56340. CrossRef Medline

Branco T, Staras K, Darcy KJ, Goda Y (2008) Local dendritic activity sets release probability at hippocampal synapses. Neuron 59:475-485. CrossRef Medline

Burrone J, O’Byrne M, Murthy VN (2002) Multiple forms of synaptic plasticity triggered by selective suppression of activity in individual neurons. Nature 420:414-418. CrossRef Medline

Cabanac M (2006) Adjustable set point: to honor Harold T. Hammel. J Appl Physiol 100:1338-1346. CrossRef Medline

Campbell LW, Hao SY, Thibault O, Blalock EM, Landfield PW (1996) Aging changes in voltage-gated calcium currents in hippocampal CA1 neurons. J Neurosci 16:6286-6295. Medline

Chouhan AK, Zhang J, Zinsmaier KE, Macleod GT (2010) Presynaptic mitochondria in functionally different motor neurons exhibit similar affinities for $\mathrm{Ca}^{2+}$ but exert little influence as $\mathrm{Ca}^{2+}$ buffers at nerve firing rates in situ. J Neurosci 30:1869-1881. CrossRef Medline

Davis GW (2006) Homeostatic control of neural activity: from phenomenology to molecular design. Annu Rev Neurosci 29:307-323. CrossRef Medline

Davis GW (2013) Homeostatic signaling and the stabilization of neural function. Neuron 80:718-728. CrossRef Medline

Davis GW, DiAntonio A, Petersen SA, Goodman CS (1998) Postsynaptic PKA controls quantal size and reveals a retrograde signal that regulates presynaptic transmitter release in Drosophila. Neuron 20:305-315. CrossRef Medline

Dumas TC, Foster TC (1995) Developmental increase in CA3-CA1 presyn- 
aptic function in the hippocampal slice. J Neurophysiol 73:1821-1828. Medline

Foster TC (2007) Calcium homeostasis and modulation of synaptic plasticity in the aged brain. Aging Cell 6:319-325. CrossRef Medline

Foster TC, Barnes CA, Rao G, McNaughton BL (1991) Increase in perforant path quantal size in aged F-344 rats. Neurobiol Aging 12:441-448. Medline

Frank CA (2013) Homeostatic plasticity at the Drosophila neuromuscular junction. Neuropharmacology. Advance online publication. Retrieved October 1, 2013. doi:10.1016/j.neuropharm.2013.06.015. CrossRef Medline

Frank CA, Kennedy MJ, Goold CP, Marek KW, Davis GW (2006) Mechanisms underlying the rapid induction and sustained expression of synaptic homeostasis. Neuron 52:663-677. CrossRef Medline

Frank CA, Pielage J, Davis GW (2009) A presynaptic homeostatic signaling system composed of the Eph receptor, ephexin, Cdc42, and CaV2.1 calcium channels. Neuron 61:556-569. CrossRef Medline

Gordon MD, Scott K (2009) Motor control in a Drosophila taste circuit. Neuron 61:373-384. CrossRef Medline

Hori N, Hirotsu I, Davis PJ, Carpenter DO (1992) Long-term potentiation is lost in aged rats but preserved by calorie restriction. Neuroreport 3:1085-1088. CrossRef Medline

Kelly SS (1978) The effect of age on neuromuscular transmission. J Physiol 274:51-62. Medline

Landfield PW, McGaugh JL, Lynch G (1978) Impaired synaptic potentiation processes in the hippocampus of aged, memory-deficient rats. Brain Res 150:85-101. Medline

Marder E, Prinz AA (2002) Modeling stability in neuron and network function: the role of activity in homeostasis. Bioessays 24:1145-1154. CrossRef Medline

Marder E, Prinz AA (2003) Current compensation in neuronal homeostasis. Neuron 37:2-4. CrossRef Medline

Marie B, Pym E, Bergquist S, Davis GW (2010) Synaptic homeostasis is consolidated by the cell fate gene gooseberry, a Drosophila pax3/7 homolog. J Neurosci 30:8071-8082. CrossRef Medline

McCabe AK, Chisholm SL, Picken-Bahrey HL, Moody WJ (2006) The selfregulating nature of spontaneous synchronized activity in developing mouse cortical neurones. J Physiol 577:155-167. CrossRef Medline

Moody WJ, Bosma MM (2005) Ion channel development, spontaneous activity, and activity-dependent development in nerve and muscle cells. Physiol Rev 85:883-941. CrossRef Medline

Morrison JH, Baxter MG (2012) The ageing cortical synapse: hallmarks and implications for cognitive decline. Nat Rev Neurosci 13:240-250. CrossRef Medline

Müller M, Davis GW (2012) Transsynaptic control of presynaptic $\mathrm{Ca}^{2+}$ influx achieves homeostatic potentiation of neurotransmitter release. Curr Biol 22:1102-1108. CrossRef Medline

Paradis S, Sweeney ST, Davis GW (2001) Homeostatic control of presynaptic release is triggered by postsynaptic membrane depolarization. Neuron 30:737-749. CrossRef Medline

Petersen SA, Fetter RD, Noordermeer JN, Goodman CS, DiAntonio A
(1997) Genetic analysis of glutamate receptors in Drosophila reveals a retrograde signal regulating presynaptic transmitter release. Neuron 19: 1237-1248. CrossRef Medline

Rabinowitch I, Segev I (2008) Two opposing plasticity mechanisms pulling a single synapse. Trends Neurosci 31:377-383. CrossRef Medline

Rawson JM, Kreko T, Davison H, Mahoney R, Bokov A, Chang L, Gelfond J, Macleod GT, Eaton BA (2012) Effects of diet on synaptic vesicle release in dynactin complex mutants: a mechanism for improved vitality during motor disease. Aging Cell 11:418-427. CrossRef Medline

Robbins N (1992) Compensatory plasticity of aging at the neuromuscular junction. Exp Gerontol 27:75-81. Medline

Rosenmund C, Stevens CF (1996) Definition of the readily releasable pool of vesicles at hippocampal synapses. Neuron 16:1197-1207. CrossRef Medline

Stevens CF, Williams JH (2007) Discharge of the readily releasable pool with action potentials at hippocampal synapses. J Neurophysiol 98:3221-3229. CrossRef Medline

Thiagarajan TC, Lindskog M, Tsien RW (2005) Adaptation to synaptic inactivity in hippocampal neurons. Neuron 47:725-737. CrossRef Medline

Thiagarajan TC, Lindskog M, Malgaroli A, Tsien RW (2007) LTP and adaptation to inactivity: overlapping mechanisms and implications for metaplasticity. Neuropharmacology 52:156-175. CrossRef Medline

Thibault O, Landfield PW (1996) Increase in single L-type calcium channels in hippocampal neurons during aging. Science 272:1017-1020. CrossRef Medline

Thoby-Brisson M, Simmers J (2000) Transition to endogenous bursting after long-term decentralization requires de novo transcription in a critical time window. J Neurophysiol 84:596-599. Medline

Turrigiano G (2012) Homeostatic synaptic plasticity: local and global mechanisms for stabilizing neuronal function. Cold Spring Harb Perspect Biol 4:a005736. CrossRef Medline

Turrigiano G, Abbott LF, Marder E (1994) Activity-dependent changes in the intrinsic properties of cultured neurons. Science 264:974-977. CrossRef Medline

Turrigiano G, LeMasson G, Marder E (1995) Selective regulation of current densities underlies spontaneous changes in the activity of cultured neurons. J Neurosci 15:3640-3652. Medline

Turrigiano GG, Nelson SB (2004) Homeostatic plasticity in the developing nervous system. Nat Rev Neurosci 5:97-107. Medline

Turrigiano GG, Leslie KR, Desai NS, Rutherford LC, Nelson SB (1998) Activity-dependent scaling of quantal amplitude in neocortical neurons. Nature 391:892-896. CrossRef Medline

Veng LM, Mesches MH, Browning MD (2003) Age-related working memory impairment is correlated with increases in the L-type calcium channel protein alpha1D (Cav1.3) in area CA1 of the hippocampus and both are ameliorated by chronic nimodipine treatment. Mol Brain Res 110:193202. Medline

Vitureira N, Letellier M, Goda Y (2012) Homeostatic synaptic plasticity: from single synapses to neural circuits. Curr Opin Neurobiol 22:516-521. Medline 